Selcuk Journal of Agriculture and Food Sciences

http://sjafs.selcuk.edu.tr/sjafs/index

Research Article
SJAFS

(2021) 35 (3), 229-243

e-ISSN: $2458-8377$

DOI:10.15316/SJAFS.2021.252

\title{
Determination of Effective and Specific Physical Features of Rice Varieties by Computer Vision In Exterior Quality Inspection
}

\author{
iD Ilkay CINAR ${ }^{1, *}$, ID Murat KOKLU ${ }^{1}$ \\ ${ }^{1}$ Selçuk University, Faculty of Technology, Department of Computer Engineering, Konya, Turkey
}

\begin{tabular}{l}
\hline ARTICLE INFO \\
\hline Article history: \\
Received date: 14.10 .2021 \\
Accepted date: 26.10 .2021 \\
\hline
\end{tabular}

Keywords:

Color spaces

Feature extraction

Feature selection

Image processing

Quality control

\begin{abstract}
In this study, feature extraction processes were performed based on the image processing techniques using morphological, shape and color features for five different rice varieties of the same brand. A total of 75 thousand pieces of rice grain were obtained, including 15 thousand pieces of each variety of rice. Preprocessing operations were applied to the images and made available for feature extraction. A total of 106 features were inferred from the images; 12 morphological features and 4 shape features obtained using morphological features and 90 color features obtained from five different color spaces (RGB, HSV, $\mathrm{L}^{*} \mathrm{a} * \mathrm{~b} *$ YCbCr, XYZ). In addition, for the 106 features obtained, features were selected by ANOVA, X2 and Gain Ratio tests and useful features were determined. In all tests, out of 106 features, the 5 most effective and specific features were obtained roundness, compactness, shape factor 3 , aspect ratio and eccentricity. The color features were listed in different order following these features.
\end{abstract}

\section{Introduction}

Rice is the most important product after wheat and corn when the production values of grain products worldwide are considered. Rice is a grain product that is quite rich in carbohydrates and starch. In addition, it also has great significance in human nutrition due to its economical and nutritious value. In the same time, it is also widely used in many fields of industry (Juliano 1993).

There are different quality criteria for rice varieties produced in the world. These criteria include properties of rice such as cooking properties, physical appearance, aroma and taste properties as well as productivity. From the point of view of the ultimate consumer, the first criterion that comes to mind for rice varieties sold in packages on market shelves is physical appearance (Webb 1991, Hua, Xu et al. 2021). Therefore, more technological and effective methods are needed. It is especially inefficient and take excessive time to calibrate rice and separate them within various quality criteria during production especially one considers the high production volume.

Recent studies using image processing and machine learning methods have been studied in the literature. In one of these studies, in which the projection areas of some grain products such as wheat, barley, corn, chickpeas, lentils, beans, kidney beans and soy were determined by image processing technique, the projection areas of the products in three different locations were determined. With the UTHSCSA (University of Texas Health Science Center, San Antonio) image processing program, the relationships between the projection areas were analyzed by regression analysis by obtaining feature values such as length, width and thickness of the products used in the study. As a result of the research, it was concluded that the image processing technique is sufficient for the precise determination of the projection areas of small grain products. Morphological features of 13 different wheat varieties from bread and durum wheat type with image processing technique have been evaluated using the UTHSCSA Image Tool Version 3.0 program. As a result, it has been concluded that the results of measurements obtained by hand and image processing are close and that the image processing technique can be used to determine some of the morphological features of wheat grains (Demirbas and Dursun 2007). In another study using dried tobacco leaves, a system based on machine vision techniques was developed for automatic examination of the leaves. In this system, it is aimed to analyze tobacco leaves using color, size, shape and surface texture features. Based on experimental results, it is stated that this system is a suitable route for dried tobacco leaves. Besides, it is also stated that these features of tobacco leaves can be used for automatic classification as the purpose of the next studies (Zhang and Zhang 2008).

\footnotetext{
*Corresponding author email: mkoklu@selcuk.edu.tr
} 
Aggarwal and Mohan analyzed the aspect ratio using the image processing technique for the grain quality of the rice. Rice samples from three different classes (full, semi-and broken) sold in grocery stores and priced according to their size were taken. It is aimed to examine the mixtures of these samples and determine the reference aspect ratio on the market (Aggarwal and Mohan 2010). Another study aimed to extract the morphological features of 5 pieces pasta wheat varieties called Showa, Altar 84, Altar 84-3, Dipper and Bushen with image processing technique. Five sets of features were used for linear discrimination analysis. $67.66 \%$ classification accuracy was obtained as the best result of the discrimination analysis using 11 morphological features (Farahani 2012). One of the studies was carried out using Gujarat-17 rice seeds with image analysis. It was aimed to perform quality analysis using the area, major axis length, minor axis length and eccentricity features on a certain number of samples. It is stated that the traditional quality assessment made by humans can be time consuming and expensive, and that quality analysis can be done without destruction with image analysis (Maheshwari, Jain et al. 2012). In a study on defining barley varieties, the effectiveness of determining the varieties based on the shape, color and texture features obtained from the seed image was evaluated. In the study, success rates ranging from $67 \%$ to $86 \%$ were achieved in linear distinctive analysis and artificial neural networks classifications. It has been stated that the classification results can be improved by standardizing seed images in terms of front and rear orientations and additional analyses that can be applied to wrinkled regions on the seed (Szczypiński, Klepaczko et al. 2015).

In another study on rice grains, a method for quality analysis using image processing techniques and geometric features of grains was proposed. Using MATLAB technology, the seven geometric features of rice grains (major axis length, minor axis length, eccentricity, area, orientation, perimeter, aspect ratio) were extracted from digital images and then grains belonging to certain varieties are divided into three different classes. The error ratio measuring different geometric features between the recommended method and the experimental analysis was achieved between $1.39 \%$ and $1.40 \%$ (Kaur and Singh 2015). Tin at all carried out using image processing techniques on 5 different varieties of rice specific to Myanmar, 5 morphological features were extracted for each variety. The study is stated to be realized out to develop computer-based systems in order to automatically classify rice varieties (Tin, Mon et al. 2018).

When the studies conducted in recent years are examined, it can be seen that image processing and machine vision systems are used on various grain products. In these studies, products were examined in terms of various morphological features such as quality, texture, color and size. In addition to morphological features, it also can be seen that various studies have been done using shape and color features.

\section{Materials and Methods}

In the study, a total of 75 thousand rice grain images were obtained, primarily 15 thousand for each variety (Available from: www.muratkoklu.com/datasets/Rice_Image_Dataset.ra r). The resulting images have been prepared for the feature extraction phase by undergoing various preoperations in MATLAB software. For images passed from pre-processing, 12 morphological features, 4 shape features obtained using morphological features, and with 90 color features obtained from five different color spaces, a total of 106 features were extracted (Available from: www.muratkoklu.com/datasets/Rice_MSC_Dateset.rar ). Density distribution graphs of morphological and shape features were given and the distributions of rice varieties on features were examined. Finally, 106 features obtained to reduce the data size were feature selection made by ANOVA, X2 and Gain Ratio tests and the effective features were determined. The process stages of the study are given in Figure 1.

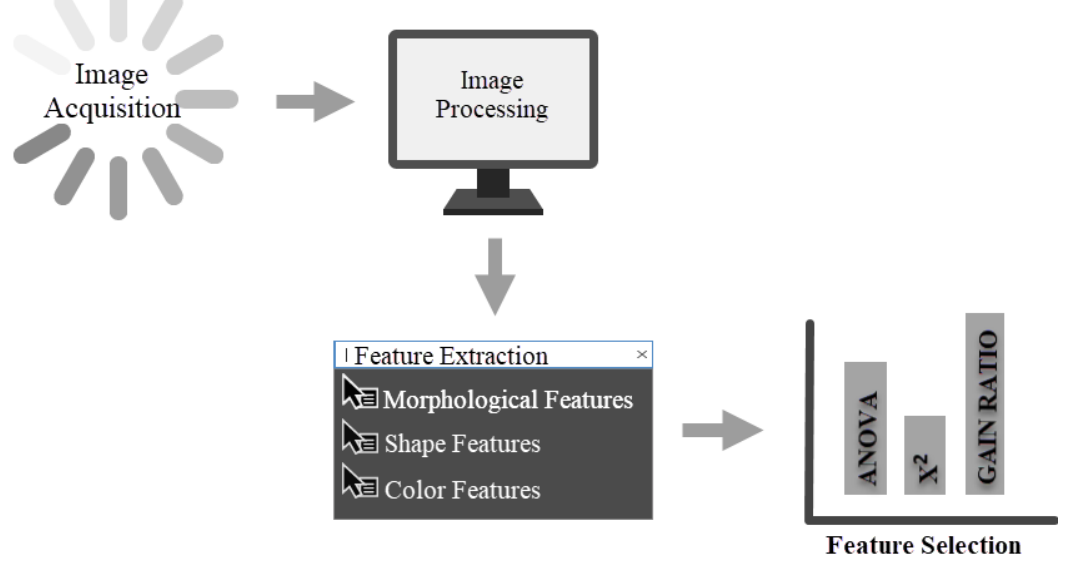

Figure 1

Process steps for the study 


\subsection{Image Acquisition}

In image processing, firstly, the image is obtained with the help of sensors or cameras and digitization pro cess is performed. In Figure 2 shows the image capture and digitization processes.

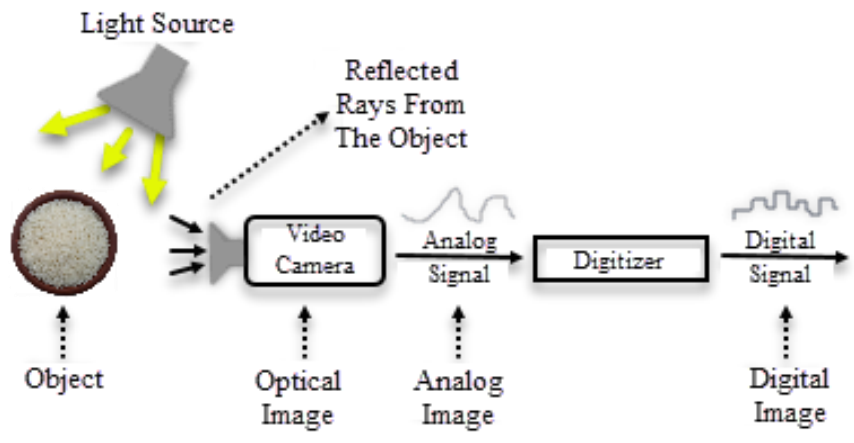

Figure 2

Capture and digitization of images

In Figure 2, reflected rays from the object illuminated by the light source are transferred to the camera. With the help of the camera, the object image is converted into analog form. The digitizer converts analog signals to digital signals, allowing the image to be processed and analyzed in a computer environment. In Figure 3, it is shown the post-digitization status of an image taken with the help of the camera.

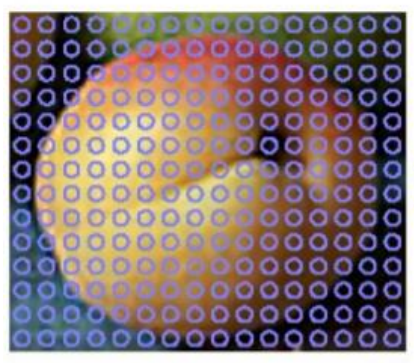

Real Image

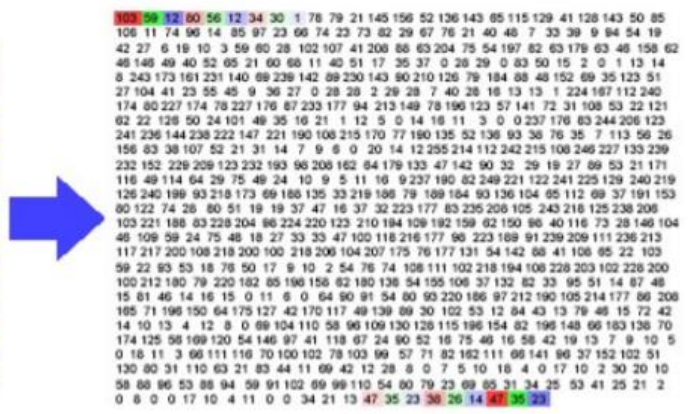

Digital Image

Figure 3

Real and digital image

In order to obtain images of rice varieties, the equipments given in Figure 4 was used. A camera with Ikegami brand CCD imaging sensor was used to acquire images. The camera has advanced sensitivity and high image quality. It displays at PAL resolution of
752(H) X582 (V). Features such as white balance and backlight correction are available. It is powered by $12 \mathrm{~V}$ DC voltage and has a power consumption below $4.5 \mathrm{~W}$ (Ikegami 2019).

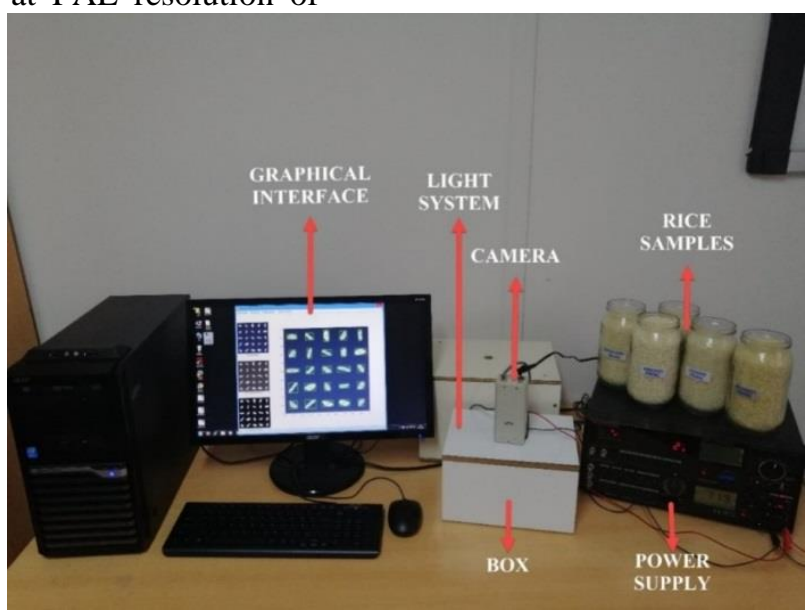

Figure 4

Equipment used to obtain images 
The camera used in the study was placed on a closed box with a lighting mechanism in it and a structure to prevent taking light from the outside environment. The box floor background is chosen black for easy processing of the image. The box dimensions are designed so that an image can be taken from an area of $14 \mathrm{~cm}$ wide and $18 \mathrm{~cm}$ length. The height of the camera to the box floor is set to $15 \mathrm{~cm}$. The images obtained from the camera were transferred to the computer and recorded (Cinar 2019).

\subsection{Image Processing}

Image processing is the process of transferring digitally acquired images to the computer environment and

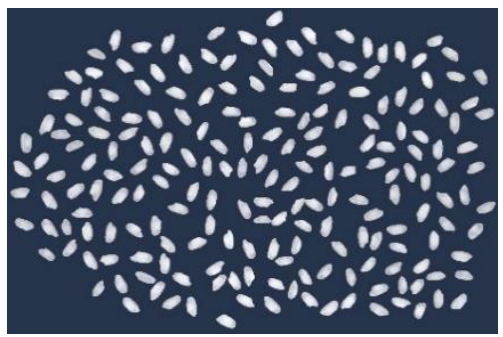

a

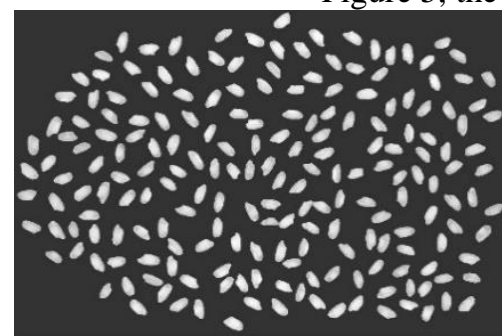

b processing them and then transmitting them to the output unit (Kwan, Mora et al. 1999). In the image processing phase, pre-processes related to images are explained in order to perform feature extraction operations in the most accurate way.

Image processing was carried out with the help of MATLAB software. Images taken from the camera were primarily converted to grayscale images. Later, with the help of otsu method, the grayscale image was converted to a binary image using the global threshold level (Otsu 1979). Unwanted objects in the obtained binary images have been removed and prepared for the feature extraction stage by applying imopen process. In Figure 5, the image pre-processing stages are given.

Figure 5

Image pre-processing stages ((a) Color Image (b) Grayscale image (c) Post-preprocessing binary image)

\subsection{Feature Extraction}

Feature extraction is the process of obtaining characteristics such as shape, texture, color and contrast from images as numerical information (Shree and Kumar 2018). In the study, 12 morphological features were extracted and 4 shape features obtained by using these morphological features were extracted. In addi-

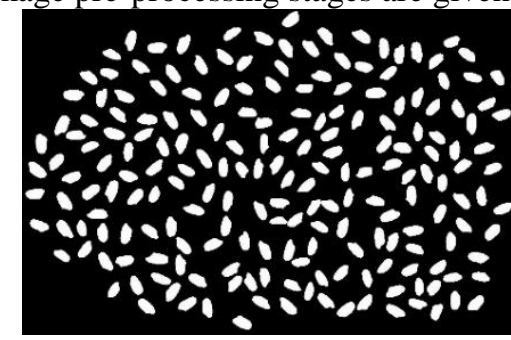

c

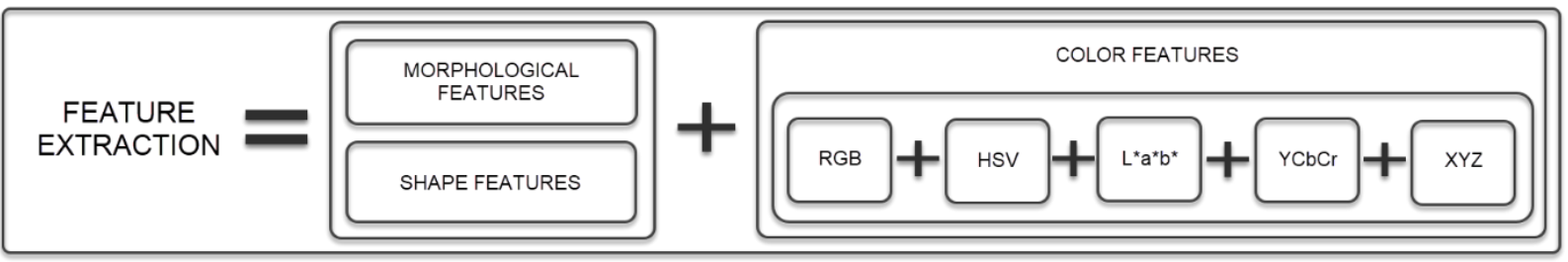

Figure 6

Process stages of feature extraction

\subsubsection{Morphological and shape features}

Morphological and shape features have been obtained using MATLAB regionprops function components (Buksh, Routh et al. 2014). The feature values

Table 1

List of morphological and shape features obtained represent the number of pixels of each grain of rice. In Table 1, A list of morphological and shape features is given.

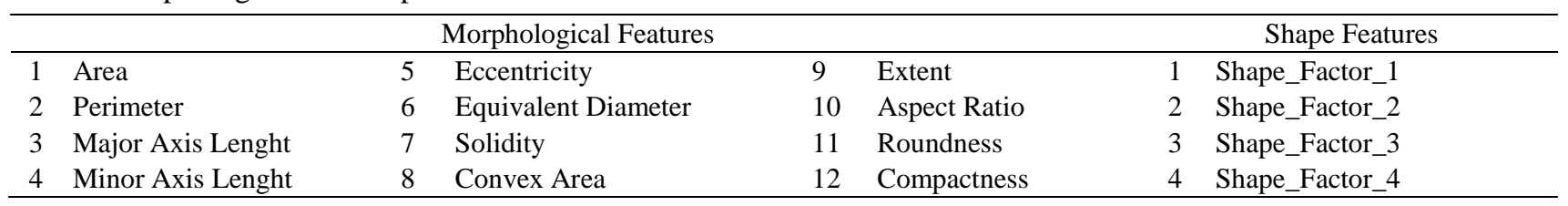

Explanations of morphological and shape features components are given below (Pazoki, Farokhi et al. 2014);

Area (A): It is the number of pixels within the boundaries of the rice grain area. tion color images, from RGB (red, green, blue) color space to HSV (hue, saturation, value), L*a*b* (L*: lightness, $a^{*}:$ red/green value, $b^{*}$ : blue/yellow value.), YCbCr (y: luminance, cb: chroma blue, cr: chroma red) and $\mathrm{XYZ}$ color spaces by performing conversion operations, a total of 90 color features were extracted from five different color spaces (Cinar 2019). In Figure 6 , the process stages of feature extraction are given. 
Minor Axis Lenght (l): It is the longest line on a grain of rice that can be drawn perpendicular to the major axis.

Eccentricity (Ec): Gives the eccentricity of the circle, which has the same moments as the region.

Equivalent Diameter (ED): It is the diameter of a circle with the same area as the area of the rice grain. The equivalent diameter was calculated according to Equation 1.

$\mathrm{ED}=\sqrt{\frac{4 \mathrm{xA}}{\pi}}$

Solidity $(S)$ : It is the ratio of pixels in the convex stem to pixels in the rice grain region. Calculated according to Equation 2.

$\mathrm{S}=\frac{\mathrm{A}}{\mathrm{CA}}$

Convex Area (CA): It is the number of pixels in the smallest convex Polygon that can accommodate the rice grain area.

Extent $(E x)$ : Bounding is the ratio of pixels in the box to pixels in the rice grain region.

Aspect Ratio $(A R)$ : It is calculated by dividing the major axis length by the minor axis length. Calculated according to Equation 3.

$\mathrm{AR}=\frac{\mathrm{L}}{1}$

Roundness (Ro): It is calculated by making use of the area and the perimeter. The Roundness was calculated according to Equation 4.

$\mathrm{Ro}=\frac{4 \mathrm{xAx} \pi}{\mathrm{P}^{2}}$

Compactness $(\mathrm{Co})$ : It is calculated by dividing the equivalent diameter by the length of the major axis. Calculated according to Equation 5.

$\mathrm{Co}=\frac{\mathrm{ED}}{\mathrm{L}}$

Shape Factor: Shape features are calculated using area, major axis and minor axis lengths from morphological features. Calculation formulas for shape factors are given below (Pazoki, Farokhi et al. 2014, Martínez, Gila et al. 2018).

Shape_Factor_1 (SF1): It is calculated by dividing the major axis length by the area. The calculation was made according to Equation 6.

$\mathrm{SF} 1=\frac{\mathrm{L}}{\mathrm{A}}$

Shape_Factor_2 (SF2): It is calculated by dividing the minor axis length by the area. Calculated according to Equation 7.

$\mathrm{SF} 2=\frac{1}{\mathrm{~A}}$
Shape_Factor_3 (SF3): The calculation was made according to Equation 8.

$\mathrm{SF} 3=\frac{\mathrm{A}}{\left(\frac{\mathrm{L}}{2}\right)^{2} \times \pi}$

Shape_Factor_4 (SF4): Calculated according to Equation 9.

$\mathrm{SF} 4=\frac{\mathrm{A}}{\frac{\mathrm{L}}{2} \times \frac{1}{2} \times \pi}$

\subsubsection{Color features}

Color images of rice grains used in the study were converted from RGB color space to HSV, L*a*b*, $\mathrm{YCbCr}$ and $\mathrm{XYZ}$ color spaces with the help of MATLAB software. Information about color spaces and conversion formulas is given below.

Color spaces: It is a mathematical representation used in defining color. Some color fields are formulated to allow people to choose colors, while others are formulated to facilitate the processing of data on machines. Color spaces are designed in three dimensions. Each pixel in the color image consists of 3 color channels (Wu and Sun 2013).

RGB Color space: RGB represents the primary colors red (R), green $(\mathrm{G})$, and blue (B). All other colors are represented by a linear combination of these three main colors (Koschan and Abidi 2008). RGB color values are expressed in a range of values from 0 to 255 . It is represented by black color values $(0,0,0)$ and white color values $(255,255,255)$. The values on the main diagonal represent the gray color values. Since RBG is considered the basic color model for image applications, it is possible to see the image on the screen without requiring any conversion (Ibraheem, Hasan et al. 2012).

HSV Color space: It consists of three parameters: hue $(\mathrm{H})$, saturation $(\mathrm{S})$, and value $(\mathrm{V})$. The hue refers to the dominant wavelength of the color and takes a value between 0-360 degrees. Saturation refers to the vitality of color. A high saturation value causes colors to be vivid, while a low saturation value causes the color to approach tones of gray. The value refers to the brightness of the color, that is, the ratio of white in it. As the brightness value gets closer to zero, the color gets closer to black tones, and otherwise to white tones (GarcíaMateos, Hernández-Hernández et al. 2015).

RGB-HSV Conversion; RGB-HSV conversion formulas are given between Equation 10 and Equation 14 (Chaudhary, Chaudhari et al. 2012, Pazoki, Farokhi et al. 2014).

$$
\begin{aligned}
& \operatorname{Max}=\operatorname{Max}(R, G, B) \\
& \operatorname{Min}=\operatorname{Min}(R, G, B) \\
& \text { V }=\operatorname{Max} \\
& \text { S }=\frac{\operatorname{Max}-\operatorname{Min}}{\operatorname{Max}}
\end{aligned}
$$


$\mathrm{H}=\left\{\begin{array}{cc}\frac{1}{6} \frac{\mathrm{G}-\mathrm{B}}{\mathrm{Max}-\mathrm{Min}}, & \mathrm{V}=\mathrm{R} \\ \frac{1}{6} \frac{\mathrm{B}-\mathrm{R}}{\operatorname{Max}-\operatorname{Min}}+\frac{1}{3}, & \mathrm{~V}=\mathrm{G} \\ \frac{1}{6} \frac{\mathrm{R}-\mathrm{G}}{\operatorname{Max}-\operatorname{Min}}+\frac{2}{3}, & \mathrm{~V}=\mathrm{B} \\ (\text { Eğger } \mathrm{H}<0 \rightarrow \mathrm{H}=\mathrm{H}+1) & \end{array}\right.$

$\mathrm{L} * \mathrm{a} * \mathrm{~b} *$ Color space: It is a color space defined by the CIE (International Commission on Illumination) and is also known as the CIELAB. It is often used for color control on objects. In this color space, colors are expressed as three values. The value $\mathrm{L} *$ refers to the paleness (0 Black, 100 white). a* value refers to red and green, $\mathrm{b} *$ value refers to yellow and blue, and axis values range from -128 to +128 (Beyaz, Ozturk et al. 2010, McGrath, Beck et al. 2017). While these values in $\mathrm{L}^{*} \mathrm{a} \mathrm{b}^{*}$ space are being designed, it is designed to be perceived by the human eye (McGrath, Beck et al. 2017).

RGB-L*a*b* Conversion; RGB-L*a*b* conversion formulas are given between Equation 15 and Equation 17 (Chaudhary, Chaudhari et al. 2012, Pazoki, Farokhi et al. 2014).

$\mathrm{L}=0.2126 \times \mathrm{R}+0.7152 \times \mathrm{G}+0.0722 \times \mathrm{B}$

$\mathrm{A}=1.4749 \times(0.2213 \times \mathrm{R}-0.3390 \times \mathrm{G}+0.1177 \times \mathrm{B})+128$

$\mathrm{B}=0.6245 \times(0.1949 \times \mathrm{R}+0.6057 \times \mathrm{G}-0.8006 \times \mathrm{B})+128$

YCbCr Color space: The YCbCr components are the brightness $(\mathrm{Y})$, blue difference chroma $(\mathrm{Cb})$, and red difference chroma $(\mathrm{Cr})$ components (Ibraheem, Hasan et al. 2012). YCbCr distinguishes color space, color and brightness information. Brightness actually gives information about the amount of light on the image, and colorfulness gives information about the amount of hue ratio on the image (Chaudhary, Chaudhari et al. 2012, Pazoki, Farokhi et al. 2014).

RGB-YCbCr Conversion; RGB-YCbCr conversion formulas are given between Equation 18 and Equation 20 (Chaudhary, Chaudhari et al. 2012, Pazoki, Farokhi et al. 2014).

$\mathrm{Y}=0.299 \times \mathrm{R}+0.587 \times \mathrm{G}+0.114 \times \mathrm{B}$

$\mathrm{Cb}=-0.168 \times \mathrm{R}-0.331 \times \mathrm{G}+0.500 \times \mathrm{B}$

$\mathrm{Cr}=0.500 \times \mathrm{R}-0.418 \times \mathrm{G}-0.081 \times \mathrm{B}$

XYZ Color space: It is the first color space defined by the CIE. Since the tristimulus values required to obtain color matching are in some cases negative, an artificial coordinate system has been developed to convert these values into positive. Thus, the tristimulus value and the $\mathrm{Y}$ color brightness value are chosen to be equivalent. XYZ consists of three components. $\mathrm{X}$ denotes red, $\mathrm{Z}$ denotes blue, and the $\mathrm{Y}$ component denotes brightness (Pratt 2001, Ibraheem, Hasan et al. 2012). The XYZ color space covers all the color values visible to humans (Mendoza, Dejmek et al. 2006).

RGB-XYZ Conversion; RGB-XYZ conversion formulas are given between Equation 21 and Equation
23 (Chaudhary, Chaudhari et al. 2012, Pazoki, Farokhi et al. 2014).

$\mathrm{X}=0.4124 \times \mathrm{R}+0.3576 \times \mathrm{G}+0.1805 \times \mathrm{B}$

$\mathrm{Y}=0.2126 \times \mathrm{R}+0.7152 \times \mathrm{G}+0.722 \times \mathrm{B}$

$\mathrm{Z}=0.0193 \times \mathrm{R}+0.1192 \times \mathrm{G}+0.9505 \times \mathrm{B}$

After the conversion process, the features of these color spaces were replicated using the regionprops function in MATLAB with data from the MeanIntensity (mean intensity value of each an image) and PixelValue (number of pixels in the each an image region) components. Using RGB, HSV, L*a*b*, YCbCr and $\mathrm{XYZ}$ color spaces, a total of 90 color features were obtained with the components of average, standard deviation, skewness, roundness, entropy and wavelet decomposition for each color channel of the color features of rice images (Arefi, Motlagh et al. 2011). The list of color features is given in Table 2 .

The components applied to the color features are described below, respectively (Arefi, Motlagh et al. 2011);

Mean $(\mathrm{Me})$ : It is the mean density value. ( $\mathrm{N}$ variable vector, represents $\mathrm{X}$ input data). It is calculated according to Equation 24.

$\mathrm{Me}=\frac{1}{\mathrm{~N}} \sum_{\mathrm{i}=1}^{\mathrm{N}} \mathrm{Xi}$

Standard Deviation (SD): Returns the standard deviation of pixel values. The standard deviation is the square root of the variance $(\mathrm{V})$. Calculation equations are given below.

$\mathrm{V}=\frac{1}{\mathrm{~N}-1} \sum_{\mathrm{i}=1}^{\mathrm{N}}(\mathrm{Xi}-\mathrm{Me})^{2}$

$\mathrm{SD}=\sqrt{\mathrm{V}}$

Skewness (Skw): Returns the skewness value of pixel values. The calculation formula for Skewness is calculated according to Equation 27.

$\mathrm{Skw}=\frac{\frac{1}{\mathrm{~N}-1} \sum_{\mathrm{i}=1}^{\mathrm{N}}(\mathrm{Xi}-\mathrm{Me})^{3}}{\mathrm{SD}^{3}}$

Kurtosis $(K u)$ : Returns the kurtosis value of pixel values. Kurtosis is calculated according to Equation 28.

$\mathrm{Ku}=\frac{\frac{1}{\mathrm{~N}-1} \sum_{\mathrm{i}=1}^{\mathrm{N}}(\mathrm{Xi}-\mathrm{Me})^{4}}{\mathrm{SD}^{4}}-3$

Entropy (E): Returns the entropy of the pixel values. Entropy is a statistical measurement used to characterize image texture. Given in Equation 29, pi represents the probability of the state $i$, while $\mathrm{m}$ represents the number of states.

$E=-\sum_{i=1}^{m} p_{i} \log _{2} p_{i}$ 
Wavelet Decomposition: Returns the wavelet decomposition level of the matrix from pixel value using

Table 2

List of features obtained from color spaces

\begin{tabular}{|c|c|c|c|c|c|c|}
\hline Color Space & Mean & $\begin{array}{l}\text { Standard } \\
\text { Deviation }\end{array}$ & Skewness & Kurtosis & Entropy & $\begin{array}{l}\text { Wavelet } \\
\text { Decomposition }\end{array}$ \\
\hline \multirow{3}{*}{ RGB } & Mean_RGB_R & StdDev_RGB_R & Skewness_RGB_R & Kurtosis_RGB_R & Entropy_RGB_R & Daub4_RGB_R \\
\hline & Mean_RGB_G & StdDev_RGB_G & Skewness_RGB_G & Kurtosis_RGB_G & Entropy_RGB_G & Daub4_RGB_G \\
\hline & Mean_RGB_B & StdDev_RGB_B & Skewness_RGB_B & Kurtosis_RGB_B & Entropy_RGB_B & Daub4_RGB_B \\
\hline \multirow{3}{*}{ HSV } & Mean_HSV_H & StdDev_HSV_H & Skewness_HSV_H & Kurtosis_HSV_H & Entropy_HSV_H & Daub4_HSV_H \\
\hline & Mean_HSV_S & StdDev_HSV_S & Skewness_HSV_S & Kurtosis_HSV_S & Entropy_HSV_S & Daub4_HSV_S \\
\hline & Mean_HSV_V & StdDev_HSV_V & Skewness_HSV_V & Kurtosis_HSV_V & Entropy_HSV_V & Daub4_HSV_V \\
\hline \multirow{3}{*}{$\mathrm{L} * \mathrm{a} * \mathrm{~b} *$} & Mean_LAB_L & StdDev_LAB_L & Skewness_LAB_L & Kurtosis_LAB_L & Entropy_LAB_L & Daub4_LAB_L \\
\hline & Mean_LAB_A & StdDev_LAB_A & Skewness_LAB_A & Kurtosis_LAB_A & Entropy_LAB_A & Daub4_LAB_A \\
\hline & Mean_LAB_B & StdDev_LAB_B & Skewness_LAB_B & Kurtosis_LAB_B & Entropy_LAB_B & Daub4_LAB_B \\
\hline \multirow{3}{*}{$\mathrm{YCbCr}$} & Mean_YCbCr_Y & StdDev_YCbCr_Y & Skewness_YCbCr_Y & Kurtosis_YCbCr_Y & Entropy_YCbCr_Y & $\begin{array}{l}\text { Daub4_- } \\
\text { YCbCr_Y }\end{array}$ \\
\hline & Mean_YCbCr_Cb & StdDev_YCbCr_Cb & Skewness_YCbCr_Cb & Kurtosis_YCbCr_Cb & Entropy__ YCbCr_Cb & $\begin{array}{l}\text { Daub4_- } \\
\text { YCbCr_Cb }\end{array}$ \\
\hline & Mean_YCbCr_Cr & StdDev_YCbCr_Cr & Skewness_YCbCr_Cr & Kurtosis_YCbCr_Cr & Entropy_YCbCr_Cr & $\begin{array}{l}\text { Daub4_- } \\
\text { YCbCr_Cr }\end{array}$ \\
\hline \multirow{3}{*}{$\mathrm{XYZ}$} & Mean_XYZ_X & StdDev_XYZ_X & Skewness_XYZ_X & Kurtosis_XYZ_X & Entropy_XYZ_X & Daub4_XYZ_X \\
\hline & Mean_XYZ_Y & StdDev_XYZ_Y & Skewness_XYZ_Y & Kurtosis_XYZ_Y & Entropy_XYZ_Y & Daub4_XYZ_Y \\
\hline & Mean_XYZ_Z & StdDev_XYZ_Z & Skewness_XYZ_Z & Kurtosis_XYZ_Z & Entropy_XYZ_Z & Daub4_XYZ_Z \\
\hline
\end{tabular}

\subsection{Feature Selection}

Feature selection is defined as the selection of the best subset that can represent the existing dataset. Feature selection is the process of selecting the best $\mathrm{K}$ pieces feature out of the $\mathrm{N}$ pieces features in the dataset by evaluating the features according to the method used (Forman 2003, Peralta, Del Río et al. 2015). In feature selection, ANOVA (Analysis of variance), X2 (Chi square) and Gain Ratio were used from commonly used tests.

ANOVA: It is a technique used to determine whether data from a different feature group has a common average. It is a feature selection technique used to determine whether differences in two or more sets of data are statistically significant (Gelman 2006, Beyaz and Ozturk 2016).

Chi square (X2): It is based on whether the difference between observed and expected frequencies is significant. It is used to test whether there is a relationship between features. As a result of the test, the features that are found to be unrelated are removed from the dataset (Liu and Setiono 1995).

Gain Ratio: The gain ratio is a non-symmetric criterion. Selects the highest rated features from among the
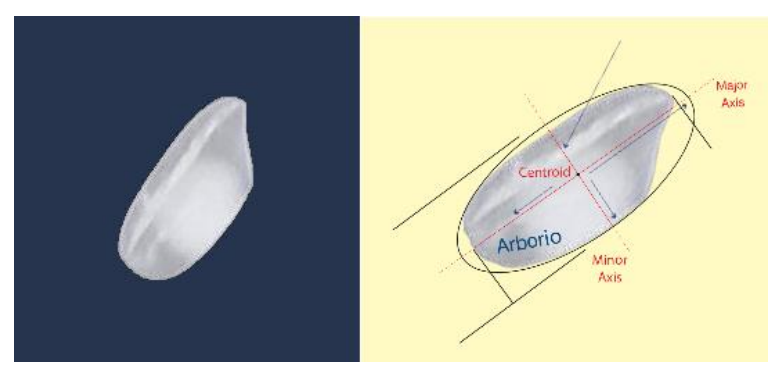

a) Arborio two-dimensional wavelet. Wavedec2 function was used and wavelet order $\mathrm{db} 4$ was selected.

features in the dataset with an average or better earnings (Quinlan 1986).

\section{Results and Discussion}

15 thousand pieces of rice grain image of each rice variety were obtained. In total, studies were carried out on the image of 75 thousand pieces rice grains. These images are pre-processed, free from unwanted substances that can be found on the image and prepared for feature extraction. Image examples of rice varieties are given in Figure 7.

A total of 106 features, including 12 morphological features, 4 shape features and 90 color features obtained from 5 different color spaces were extracted on the pre-processed images. Morphological features have been obtained using regionprops components in MATLAB software. Shape features were also obtained using these morphological features. The statistical information of the morphological and shape features, where each rice variety is evaluated separately, is given in Table 3. The values given in Table 3 are expressed as the number of pixels. The density distribution graphs for the features used to extract morphological and shape features for rice varieties are given in Figure 8.

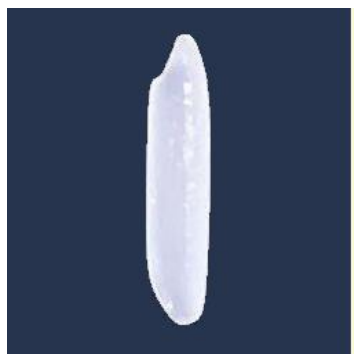

b) Basmati

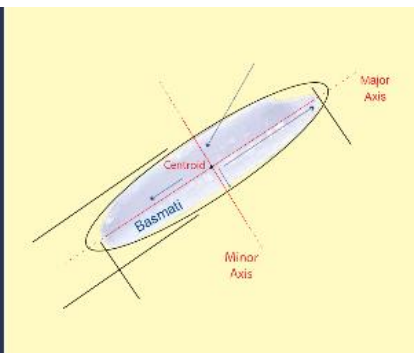



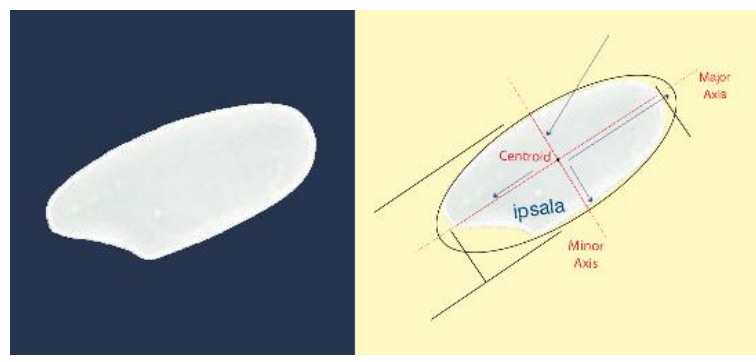

c) Ipsala

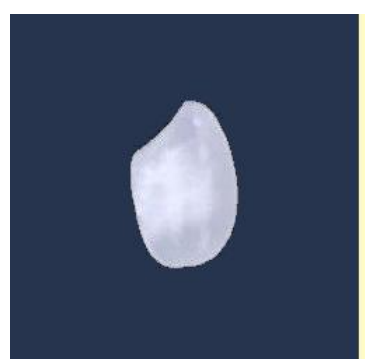

e) Karacadag
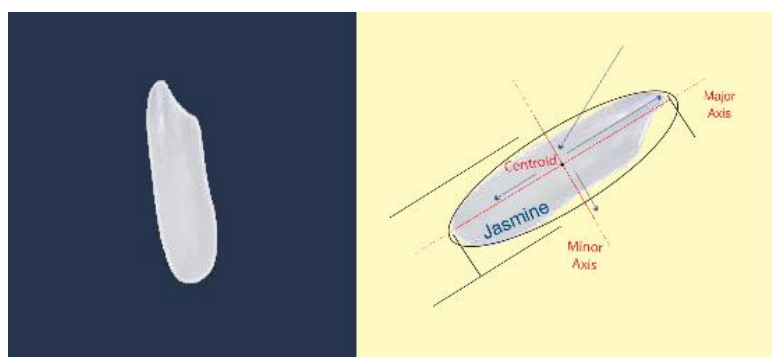

d) Jasmine

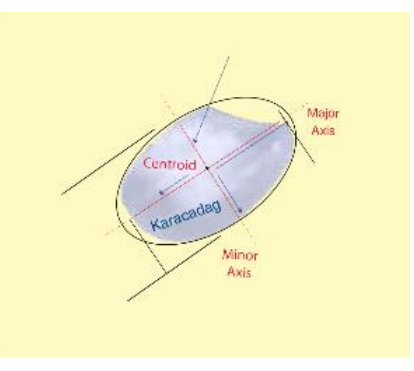

Figure 7

Image examples of rice varieties

When the density distribution graphs obtained for the 16 features of morphological and shape features are examined, it is observed that Ipsala variety differs from other rice varieties in area, minor axis length, equivalent diameter, convex area and shape factor 1 features. In the density distribution graphs of major axis length, roundness, compactness, shape factor 2, and shape factor 3, it is seen that the Karacadag variety differs from other varieties. When we look at the graphics of solidity, extent, shape factor 4 , it is seen that all varieties are mixed with each other. In addition, when the graphics of the area, perimeter, major axis length, minor axis length, equivalent diameter, convex area, shape factor 1, and shape factor 2 features are examined, it is seen that Jasmine variety is divided into two regions in terms of these features.

RGB, HSV, L*a*b, YCbCr and XYZ color spaces were used for color features. Conversion operations to other color spaces were performed by using pixel values for each RGB image. After the color conversion process, a total of 90 color features were obtained using 5 pieces color space data, mean, standard deviation, skewness, roundness, entropy and wavelet decomposition components.

In addition to the statistical information of morphological and shape features given for each of the rice varieties used in the study, the statistical information regarding the morphological, shape, and color features for a total of 75,000 rice grains for all varieties are given in Table 4. 
Table 3

Statistical information about morphological and shape features for rice varieties

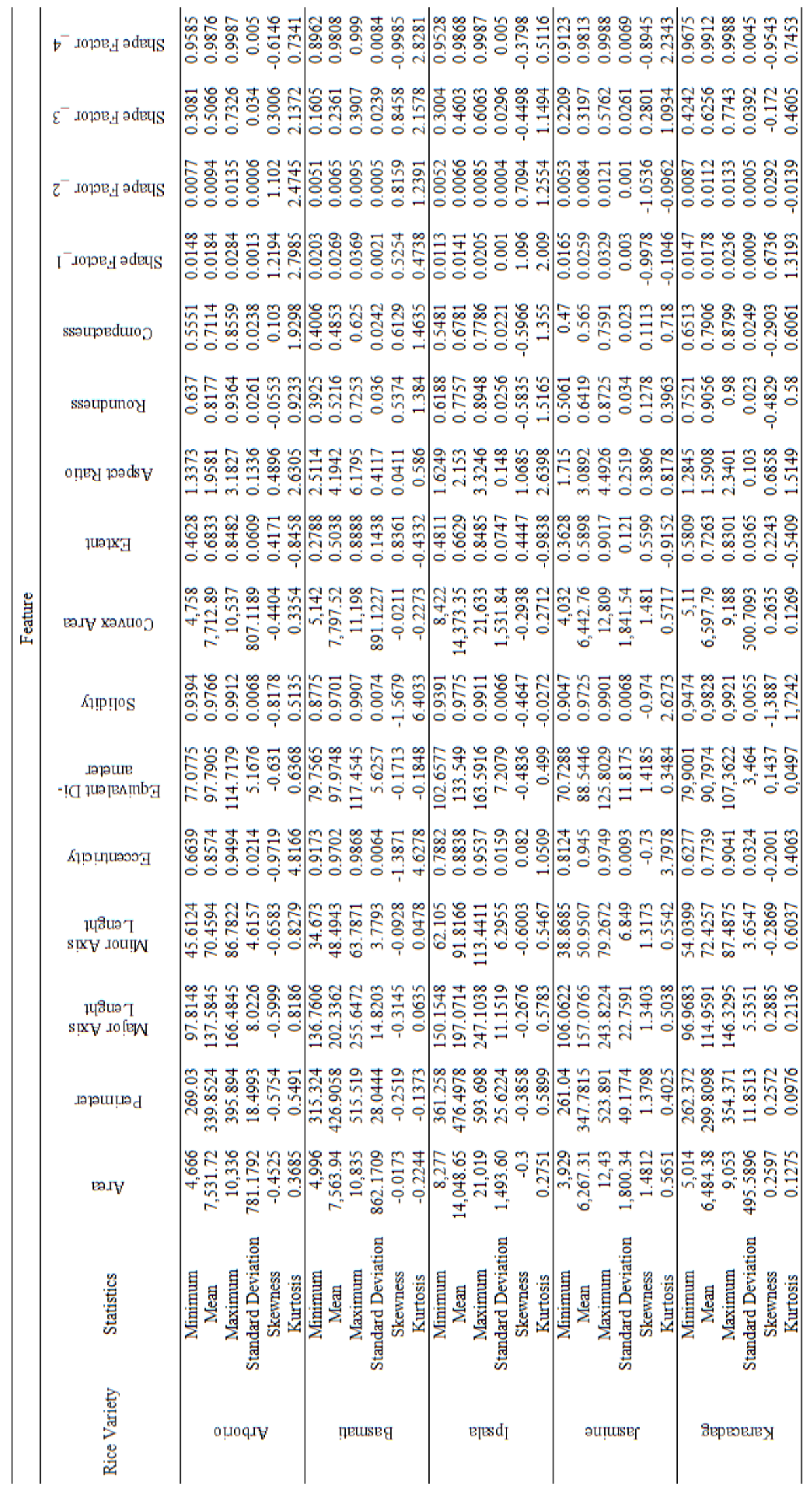


Cinar and Koklu / Selcuk J Agr Food Sci, (2021) 35 (3): 229-243
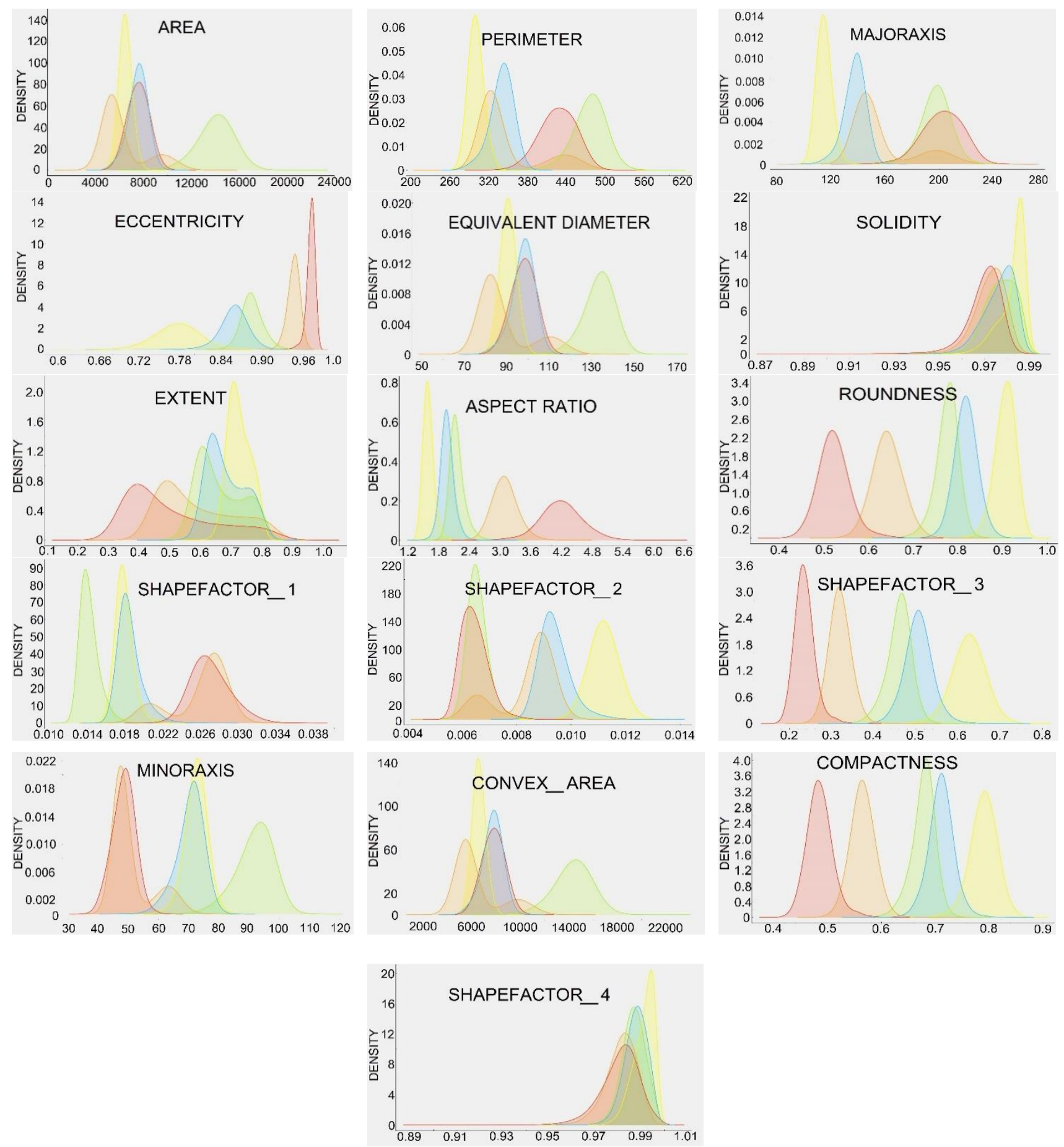

Arborio Basmati Ipsala Jasmine Karacadag

Figure 8

Density distribution graphs for morphological and shape features of rice varieties 
Cinar and Koklu / Selcuk J Agr Food Sci, (2021) 35 (3): 229-243

Table 4

Statistical information on morphological, shape and color features for all varieties

\begin{tabular}{|c|c|c|c|}
\hline 惫 & 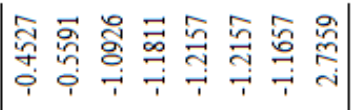 & 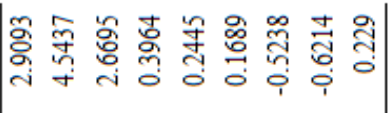 & 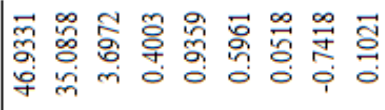 \\
\hline 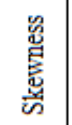 & 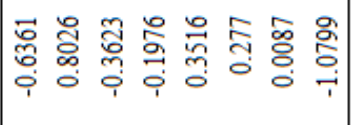 & 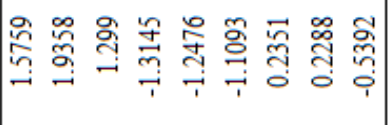 & 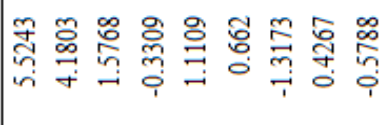 \\
\hline 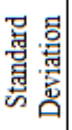 & 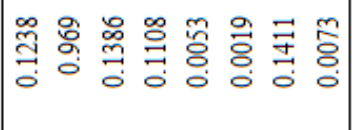 & 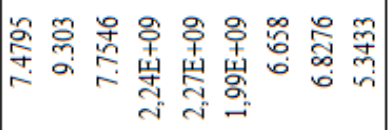 & 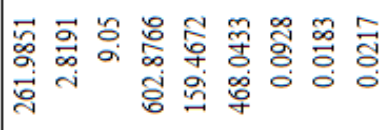 \\
\hline 買 & 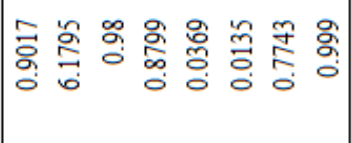 & 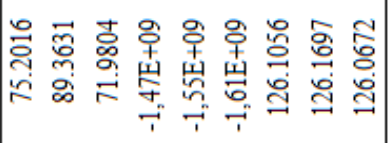 & 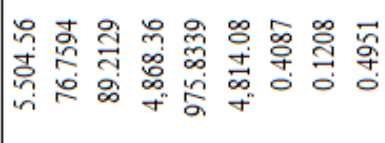 \\
\hline 䄈 & 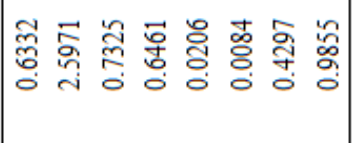 & 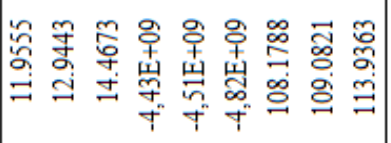 & 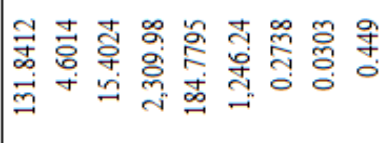 \\
\hline 慁 & 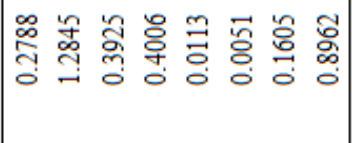 & 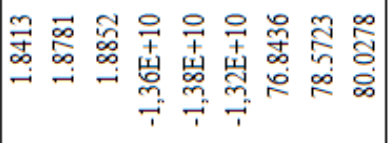 & 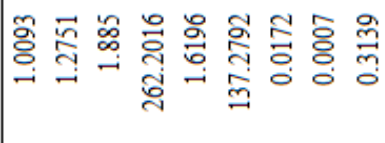 \\
\hline 莺 & 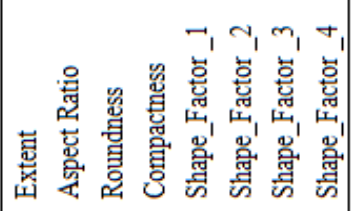 & 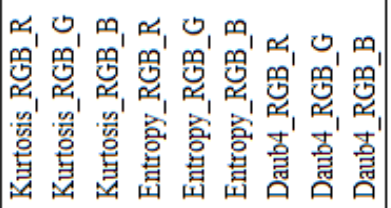 & 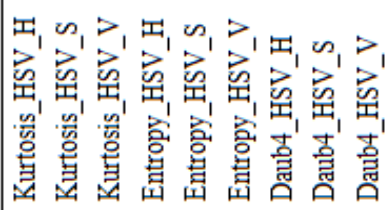 \\
\hline 㓊 & 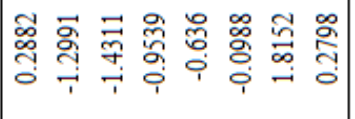 & 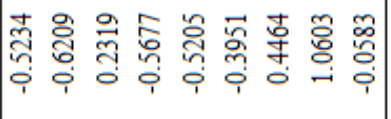 & 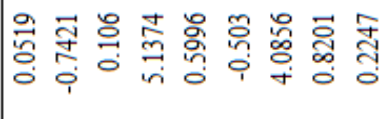 \\
\hline 总 & 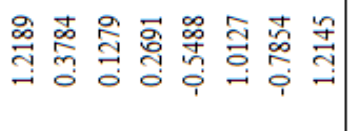 & 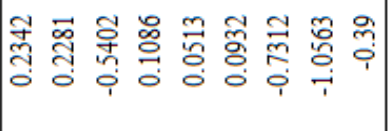 & 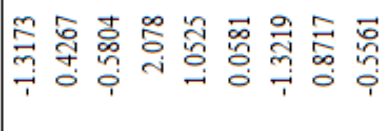 \\
\hline 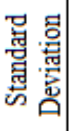 & 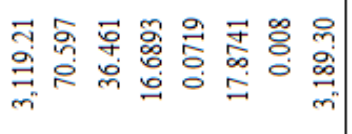 & 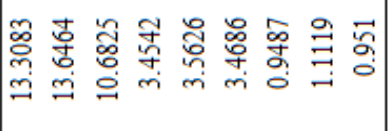 & 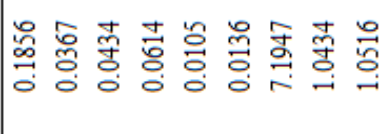 \\
\hline 買 & 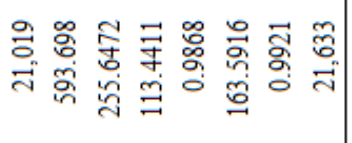 & 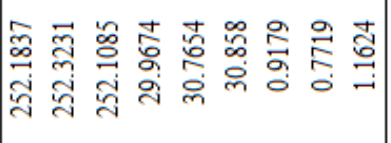 & 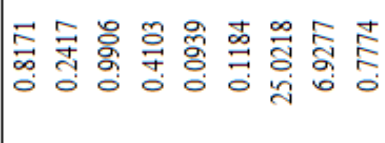 \\
\hline 急 & 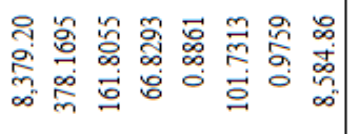 & 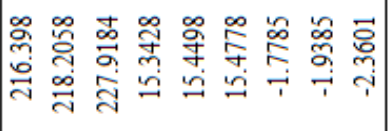 & 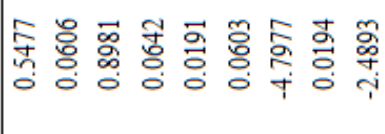 \\
\hline 買 & 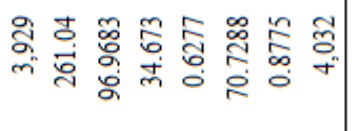 & 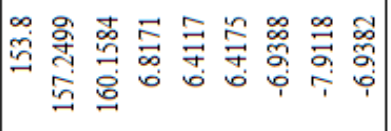 & 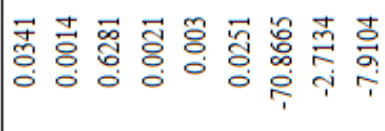 \\
\hline 节 & 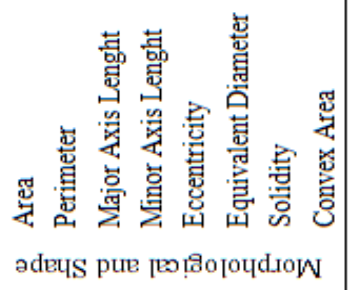 & 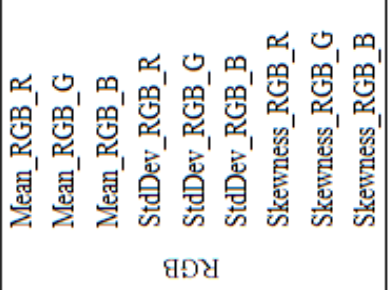 & 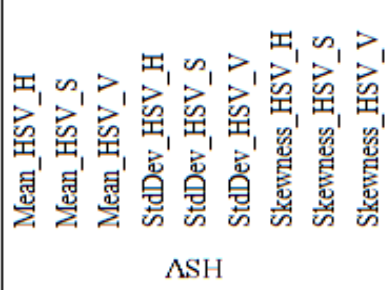 \\
\hline
\end{tabular}


Table 4 (Continued)

Statistical information on morphological, shape and color features for all varieties

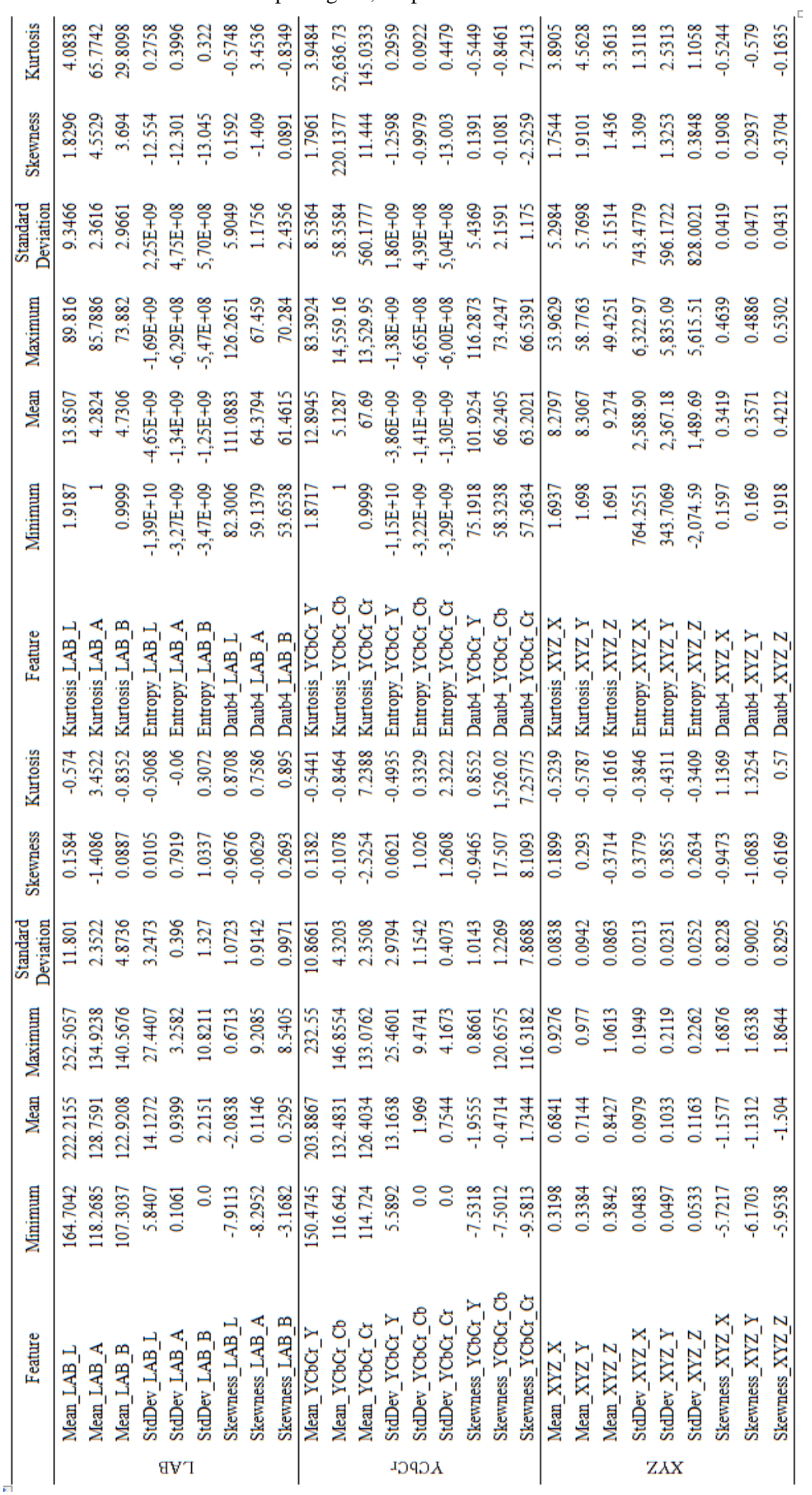


Finally, among a total of 106 features of morphological, shape, and color features, the importance order of the effective features was determined by selecting the features with ANOVA, X2, and Gain Ratio tests. The importance order of the effective features obtained from the tests is given in Table 5 .

Table 5

Importance order of effective features obtained by ANOVA, X2 and Gain Ratio tests

\begin{tabular}{|c|c|c|c|c|c|c|c|c|c|c|c|c|c|c|c|}
\hline Features & 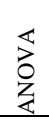 & $\tilde{x}$ & 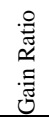 & Features & $\begin{array}{l}\longleftarrow \\
\gtrsim \\
\vdots \\
z\end{array}$ & $\tilde{x}$ & 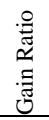 & Features & $\begin{array}{l}\nwarrow \\
\vdots \\
Z \\
2\end{array}$ & $\tilde{x}$ & 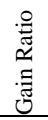 & Features & $\begin{array}{l}\varangle \\
\gtrsim \\
Z \\
Z\end{array}$ & $\tilde{x}$ & ڤ્气 \\
\hline Roundness & 1 & 1 & 1 & Entropy_YCbCr_Cb & 28 & 54 & 25 & Daub4_RGB_G & 55 & 57 & 50 & Extent & 82 & 92 & 72 \\
\hline Compactness & 2 & 2 & 2 & StdDev_HSV_V & 29 & 15 & 27 & Daub4_YCbCr_Y & 56 & 61 & 56 & Entropy_XYZ_Y & 83 & 93 & 8 \\
\hline Shape_Factor_3 & 3 & 3 & 3 & StdDev_LAB_L & 30 & 19 & 30 & Mean_RGB_G & 57 & 58 & 51 & Skewness_XYZ_X & 84 & 96 & 96 \\
\hline Aspect Ratio & 4 & 4 & 4 & StdDev_YCbCr_Y & 31 & 21 & 32 & Mean_YCbCr_Y & 58 & 62 & 57 & Skewness_YCbCr_Cb & 85 & 75 & 70 \\
\hline Eccentricity & 5 & 5 & 5 & StdDev_RGB_G & 32 & 22 & 33 & Daub4_XYZ_X & 59 & 65 & 60 & Daub4_XYZ_Z & 86 & 84 & 0 \\
\hline Minor Axis Lenght & 6 & 7 & 9 & StdDev_RGB_B & 33 & 20 & 34 & Mean_XYZ_X & 60 & 66 & 61 & Mean_XYZ_Z & 87 & 86 & 91 \\
\hline Entropy_LAB_B & 7 & 31 & 13 & StdDev_RGB_R & 34 & 16 & 31 & Kurtosis_HSV_V & 61 & 25 & 42 & Daub4_RGB_B & 88 & 87 & 92 \\
\hline Entropy_RGB_R & 8 & 30 & 24 & StdDev_LAB_B & 35 & 29 & 40 & StdDev_XYZ_X & 62 & 47 & 63 & Mean_RGB_B & 89 & 88 & 93 \\
\hline Entropy_YCbCr_Cr & 9 & 24 & 23 & Entropy_XYZ_X & 36 & 68 & 38 & Skewness_RGB_G & 63 & 69 & 75 & Entropy_XYZ_Z & 90 & 95 & 94 \\
\hline Shape_Factor_2 & 10 & 9 & 7 & Kurtosis_RGB_R & 37 & 35 & 55 & StdDev_XYZ_Y & 64 & 49 & 64 & Solidity & 91 & 97 & 100 \\
\hline Daub4_HSV_H & 11 & 71 & 66 & StdDev_YCbCr_Cb & 38 & 28 & 37 & Skewness_LAB_L & 65 & 72 & 76 & Shape_Factor_4 & 92 & 99 & 102 \\
\hline Mean_HSV_H & 12 & 70 & 65 & Daub4_RGB_R & 39 & 51 & 45 & Skewness_LAB_A & 66 & 56 & 69 & Mean_YCbCr_Cr & 93 & 81 & 84 \\
\hline Shape_Factor_1 & 13 & 6 & 8 & Mean_RGB_R & 40 & 52 & 47 & Skewness_RGB_R & 67 & 80 & 79 & Daub4_YCbCr_Cr & 94 & 82 & 85 \\
\hline Entropy_LAB_A & 14 & 38 & 26 & Kurtosis_RGB_G & 41 & 26 & 41 & Skewness_YCbCr_Y & 68 & 78 & 80 & Skewness_XYZ_Z & 95 & 94 & 98 \\
\hline Entropy_YCbCr_Y & 15 & 34 & 21 & Kurtosis_LAB_L & 42 & 32 & 46 & Skewness_LAB_B & 69 & 74 & 71 & StdDev_YCbCr_Cr & 96 & 90 & 87 \\
\hline Entropy_LAB_L & 16 & 36 & 20 & Kurtosis_YCbCr_Y & 43 & 33 & 48 & Skewness_HSV_V & 70 & 67 & 73 & Entropy_HSV_H & 97 & 101 & 77 \\
\hline Entropy_RGB_G & 17 & 37 & 19 & Mean_HSV_S & 44 & 18 & 36 & Kurtosis_XYZ_Z & 71 & 50 & 62 & Skewness_HSV_H & 98 & 102 & 99 \\
\hline Major Axis Lenght & 18 & 8 & 6 & Daub4_HSV_S & 45 & 17 & 35 & Kurtosis_RGB_B & 72 & 53 & 68 & Kurtosis_HSV_S & 99 & 89 & 89 \\
\hline Area & 19 & 45 & 17 & Kurtosis_XYZ_Y & 46 & 23 & 39 & Skewness_HSV_S & 73 & 73 & 74 & Kurtosis_LAB_B & 100 & 91 & 97 \\
\hline Convex Area & 20 & 42 & 16 & Kurtosis_XYZ_X & 47 & 27 & 43 & Skewness_XYZ_Y & 74 & 85 & 88 & StdDev_HSV_H & 101 & 100 & 95 \\
\hline Equivalent Diameter & 21 & 44 & 18 & StdDev_HSV_S & 48 & 48 & 44 & Daub4_HSV_V & 75 & 76 & 81 & Kurtosis_LAB_A & 102 & 103 & 103 \\
\hline Perimeter & 22 & 12 & 10 & StdDev_LAB_A & 49 & 43 & 49 & Mean_HSV_V & 76 & 77 & 82 & Kurtosis_HSV_H & 103 & 104 & 105 \\
\hline Daub4_LAB_B & 23 & 14 & 15 & Daub4_LAB_L & 50 & 59 & 52 & Entropy_HSV_S & 77 & 55 & 67 & Skewness_YCbCr_Cr & 104 & 105 & 104 \\
\hline Mean_LAB_B & 24 & 13 & 14 & Mean_LAB_L & 51 & 60 & 53 & Entropy_HSV_V & 78 & 83 & 86 & Kurtosis_YCbCr_Cr & 105 & 106 & 106 \\
\hline Daub4_YCbCr_Cb & 25 & 11 & 12 & Daub4_XYZ_Y & 52 & 63 & 58 & Mean_LAB_A & 79 & 39 & 28 & Kurtosis_YCbCr_Cb & 106 & 98 & 101 \\
\hline Mean_YCbCr_Cb & 26 & 10 & 11 & Mean_XYZ_Y & 53 & 64 & 59 & Daub4_LAB_A & 80 & 40 & 29 & & & & \\
\hline Entropy_RGB_B & 27 & 41 & 22 & StdDev_XYZ_Z & 54 & 46 & 54 & Skewness_RGB_B & 81 & 79 & 83 & & & & \\
\hline
\end{tabular}

The results from the ANOVA test show that morphological and shape features are the majority among the 10 most effective features. The color features contained within the first 30 features are components derived from $\mathrm{L}^{*} \mathrm{a} * \mathrm{~b} * \mathrm{HSV}, \mathrm{RGB}$, and $\mathrm{YCbCr}$ color spaces. It is also observed that components derived using the XYZ color space do not exist among the first 35 effective features.

In the X2 test, it is observed that all but one of the 10 most effective features are morphological and shape features. The color features contained within the first 30 features are components derived from $\mathrm{L}^{*} \mathrm{a} * \mathrm{~b} *$, HSV, RGB, and YCbCr color spaces. Only two of the components derived using the XYZ color space are among the first 30 effective features.

When looking at the Gain Ratio test results, it is seen that all 10 most effective features consist of morphological and shape features. The color features contained within the first 30 features are components derived from $\mathrm{L}^{*} \mathrm{a} \mathrm{b}^{*}$, $\mathrm{HSV}, \mathrm{RGB}$, and $\mathrm{YCbCr}$ color spaces. Components derived using the XYZ color space are not included among the first 37 effective features.

Finally, when the ANOVA, X2 and Gain Ratio test results are evaluated together, it is observed that the ranking has not changed in terms of the top 5 effective features. In all tests, roundness, compactness, shape factor 3 , aspect ratio and eccentricity features were obtained as the 5 most effective features.

\section{Conclusions}

A database can be created for the features of using the rice varieties mentioned in the paper. This database can be made available to the relevant sector in the field of agriculture. In addition, information such as the determination of rice varieties, morphological features etc. can be accessed instantly. Furthermore, by increasing the number of rice varieties, database can be extended. 
Using the data obtained, an automated and moving image-capturing system can be designed to distinguish rice species, and a machine can be designed to perform operations such as calibration or separation of undesirable substances from varieties.

With the 106 features used in the study, feature extractions can be performed on other varieties of rice. Automatic classification processes of varieties can be performed by using these features. In addition, the effects of effective features on the success of classification can be compared with the ANOVA, X2 and Gain Ratio tests, where effective features are determined.

Classification studies can be done with artificial intelligence methods and new algorithms. The data obtained can be applied to other agricultural products that have not been studied in the literature. Furthermore, by increasing the number of features, a greater number of feature extractions can be realized.

\section{Acknowledgements}

We would like to thank Selcuk University Scientific Research Coordinator for their support.

This research was produced from Ilkay CINAR's published Master thesis (Cinar 2019).

\section{References}

Aggarwal AK and Mohan R (2010). "Aspect ratio analysis using image processing for rice grain quality." International Journal of Food Engineering 6(5).DOI: 10.2202/1556-3758.1788.

Arefi A, Motlagh AM and Teimourlou RF (2011). "Wheat class identification using computer vision system and artificial neural networks." International Agrophysics 25(4): 319-325.

Beyaz A. and Ozturk R (2016). "Identification of olive cultivars using image processing techniques." Turkish Journal of Agriculture and Forestry 40(5): 671683.DOI: 10.3906/tar-1504-95.

Beyaz A, Ozturk R and Turker U (2010). "Assessment of mechanical damage on apples with image analysis." Journal: Food, Agriculture \& Environment (JFAE) 8(3\&4): 476-480.

Buksh R, Routh S, Mitra P, Banik S, Mallik A and Gupta SD (2014). "MATLAB based image editing and color detection." International Journal of Scientific Research Publications 4(1): 1-6.

Chaudhary P, Chaudhari AK, Cheeran A and Godara S (2012). "Color transform based approach for disease spot detection on plant leaf." International journal of computer science telecommunications 3(6): 65-70.

Cinar I. (2019). Classification of rice varieties using artificial intelligence techniques. Master Master Selcuk University.
Demirbas H and Dursun I (2007). "Determination of some physical properties of wheat grains by using image analysis." Journal of Agricultural Sciences.

Farahani L (2012). "Discrimination of some cultivars of durum wheat (Triticum durum Desf.) using image analysis." International Research Journal of Applied Basic Sciences 3(7): 1375-1380.

Forman G (2003). "An extensive empirical study of feature selection metrics for text classification." Journal of machine learning research 3(Mar): 12891305.

García-Mateos G, Hernández-Hernández J, Escarabajal-Henarejos D, Jaén-Terrones $\mathrm{S}$ band MolinaMartínez J (2015). "Study and comparison of color models for automatic image analysis in irrigation management applications." Agricultural Water Management 151: 158-166.DOI: 10.1016/j.agwat.2014.08.010.

Gelman A (2006). "Prior distributions for variance parameters in hierarchical models (comment on article by Browne and Draper)." Bayesian analysis 1(3): 515-534.

Hua S, Xu M, Xu Z and Ye H (2021). "Quantitative Evaluation of Leaf Morphology with Different Rice Genotypes Based on Image Processing." Mathematical Problems in Engineering 2021.DOI: 10.1155/2021/6620636.

Ibraheem NA, Hasan MM, Khan RZ and Mishra PK (2012). "Understanding color models: a review." ARPN Journal of science technology 2(3): 265-275.

Ikegami (2019). Retrieved 14 March 2019, from https://www.ikegami.com.

Juliano BO (1993). Rice in human nutrition, Int. Rice Res. Inst.

Kaur S and Singh D (2015). "Geometric feature extraction of selected rice grains using image processing techniques." International Journal of Computer Applications 124(8).

Koschan A and Abidi M (2008). Color spaces and color distances. Digital Color Image Processing, Wiley. 1: 37-44.

Kwan AK, Mora C and Chan H (1999). "Particle shape analysis of coarse aggregate using digital image processing." Cement Concrete Research 29(9): 1403-1410.DOI: 10.1016/S0008-8846(99)00105-2.

Liu H and Setiono R (1995). Chi2: Feature selection and discretization of numeric attributes. Proceedings of 7th IEEE International Conference on Tools with Artificial Intelligence, IEEE.DOI: 10.1109/TAI.1995.479783.

Maheshwari CV, Jain KR and Modi CK (2012). "Nondestructive quality analysis of Indian Gujarat-17 Oryza sativa SSP Indica (Rice) using image processing." International Journal of Computational Engineering Science 2: 48-54.

Martínez SS, Gila DM, Beyaz A, Ortega JG and García JG (2018). "A computer vision approach based on 
endocarp features for the identification of olive cultivars." Computers Electronics in Agriculture 154: 341-346. DOI: 10.1016/j.compag.2018.09.017.

McGrath JR, Beck M and Hill Jr ME (2017). "Replicating Red: Analysis of ceramic slip color with CIELAB color data." Journal of Archaeological Science: $\quad$ Reports 14: 432-438.DOI: 10.1016/j.jasrep.2017.06.020.

Mendoza F, Dejmek P and Aguilera JM (2006). "Calibrated color measurements of agricultural foods using image analysis." Postharvest Biology Technology 41(3): 285-295. DOI: 10.1016/j.postharvbio.2006.04.004.

Otsu N (1979). "A threshold selection method from gray-level histograms." IEEE transactions on systems, man, cybernetics 9(1): 62-66.

Pazoki A, Farokhi F and Pazoki Z (2014). "Classification of rice grain varieties using two Artificial Neural Networks (MLP and Neuro-Fuzzy)." The Journal of Animal Plant Sciences 24(1): 336-343.

Peralta D, Del Río S, Ramírez-Gallego S, Triguero I, Benitez JM and Herrera F (2015). "Evolutionary feature selection for big data classification: A mapreduce approach." Mathematical Problems in Engineering 2015.DOI: 10.1155/2015/246139.

Pratt WK (2001). "Digital Image Processing: PIKS Inside, John Wiley and Sons." Inc., New York.
Quinlan JR (1986). "Induction of decision trees." Machine learning 1(1): 81-106.DOI: 10.1007/BF00116251.

Shree NV and Kumar T (2018). "Identification and classification of brain tumor MRI images with feature extraction using DWT and probabilistic neural network." Brain informatics 5(1): 23-30.DOI: 10.1007/s40708-017-0075-5.

Szczypiński PM, Klepaczko A and Zapotoczny P (2015). "Identifying barley varieties by computer vision." Computers Electronics in Agriculture 110: 1-8.DOI: 10.1016/j.compag.2014.09.016.

Tin MM, Mon KL, Win EP and Hlaing SS (2018). Myanmar Rice Grain Classification Using Image Processing Techniques. International Conference on Big Data Analysis and Deep Learning Applications, Springer.DOI: 10.1007/978-981-13-08697_36.

Webb B (1991). Rice quality and grades. Rice, Springer: 508-538.DOI: 10.1007/978-1-4899-3754-4_16.

Wu D and Sun DW (2013). "Colour measurements by computer vision for food quality control-A review." Trends in Food Science \& Technology 29(1): 5-20.DOI: 10.1016/j.tifs.2012.08.004.

Zhang X and Zhang F (2008). Images features extraction of tobacco leaves. 2008 Congress on Image and Signal Processing, IEEE.DOI: 10.1109/CISP.2008.88. 